\title{
Development of a New Image-Guided Prostate Biopsy System
}

\author{
Jianchao Zeng ${ }^{1}$, Ariela Sofer ${ }^{2}$, and Seong K. Mun ${ }^{1}$ \\ ${ }^{1}$ ISIS Center, Department of Radiology \\ Georgetown University Medical Center, Washington, DC, USA \\ zeng@georgetown.edu, http://www.simulation.georgetown.edu \\ ${ }^{2}$ Department of Systems Engineering and Operations Research \\ George Mason University, Fairfax, VA, USA
}

\begin{abstract}
This paper presents a new image-guided prostate biopsy system under development. The system features a 3-D prostate cancer distribution atlas and new biopsy protocols optimized based on the cancer atlas using a nonlinear integer programming approach. Both the cancer atlas and the optimal protocols are being dynamically registered and superimposed onto the trans-rectal ultrasound images during live-patient biopsy procedures. Clear visual guidance will be provided to the physicians by color-coded spots on top of the ultrasound images, which represent best possible targets for biopsy. Clinical test will be performed to evaluate the system.
\end{abstract}

\section{Introduction}

Prostate cancer is usually detected clinically using trans-rectal ultrasound (TRUS) guided needle biopsy. Current ultrasound imaging systems, however, cannot provide enough information to the physicians for the best possible outcome of cancer detection. In this work, we are developing a new image-guided prostate biopsy system for significant improvement of prostate cancer detection.

\section{System Development}

\subsection{Overview of the System}

The system configuration is shown in Fig. 1. Built on top of the current TRUS imaging system, the new system will be likely to significantly improve the performance of cancer detection through the introduction of the following new features:

a. A 3-D atlas of prostate cancer distribution. The 3-D prostate cancer distribution atlas was developed by first reconstructing over 300 individual 3-D prostate models from real specimens with clinically localized cancers [1]; then dividing each individual prostate model into tiny fine zones, and checking cancer presence in each fine zone for all the models [2].

b. Optimized biopsy protocols. Optimal biopsy protocols were developed by making use of the 3-D prostate cancer distribution atlas and a physician needle insertion model [3]. A nonlinear integer program was used to determine optimal biopsy protocols on a 48-zone division of the prostate. For the same number of biopsy needles, these protocols have substantially higher detection rates than the protocols currently used by physicians. 


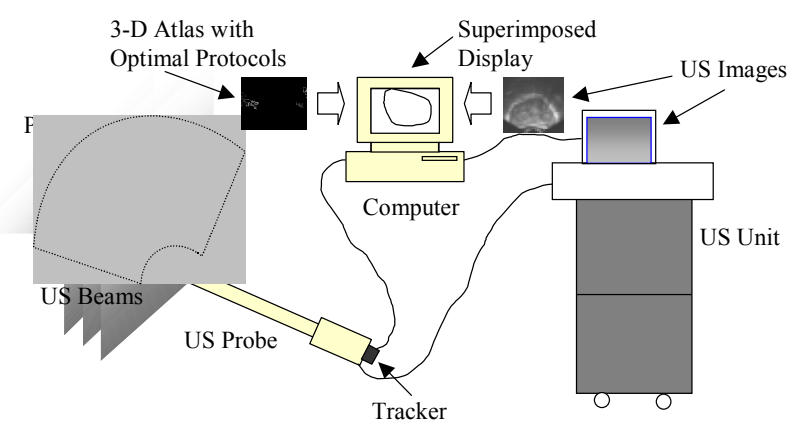

Fig. 1. Image-guided prostate biopsy system with 3-D cancer distribution atlas

c. Dynamic registration of the 3-D atlas and optimal protocols with ultrasound images. In order to dynamically register the 3-D cancer distribution atlas with highlighted optimal protocols onto the ultrasound images during the needle core biopsy, we attach a small tracking device Polhemus 3SPACE FASTRAK to the ultrasound probe to track its position and motion during biopsy. Since the developed 3-D cancer atlas is embedded in a bounding box with a number of grids, which represent the corresponding zones in the prostate gland, the registration process can be conveniently converted to the task of finding the bounding box of the individual prostate of the patient based on the ultrasound images, and aligning the two bounding boxes together in real-time.

\subsection{Segmentation of the Ultrasound Images}

In order to calculate the bounding box of the patient's prostate, we are developing an image segmentation technique to acquire the boundary of a series of transverse ultrasound images (with a few mms interval each) of the prostate. The series of transverse prostate boundaries are used to reconstruct a 3-D prostate surface model of the patient under biopsy procedure. The intersection of the ultrasound beam with the reconstructed 3-D model enables us to extract the corresponding cancer distribution information and optimized biopsy protocols from the cancer distribution atlas, and to superimpose the information on the ultrasound image as color-coded or highlighted grids.

a. Acquisition of texture statistics around the boundary of prostate capsule using a large set of transverse ultrasound image samples. For each sample image, we characterize intensity distribution around the prostate boundary. Different scales of neighborhoods of a number of selected boundary points are used to obtain simple local and regional texture information such as mean and standard deviation with Gabor filter. More texture features will also be evaluated for their applicability to the prostate ultrasound images using Gray Level Co-occurrence Matrices.

b. Boundary area extraction of the individual prostate ultrasound image with texture matching. The texture matching is guided by prior knowledge of the prostate anatomy and relative position between the prostate and the 
ultrasound probe. This process results in a focused boundary area (a belt) of the ultrasound image surrounding the prostate capsule.

c. A localized multi-scale contouring algorithm with wavelet transform is applied to the extracted boundary area. This algorithm is a coarse-to-fine process, which makes use of multi-resolution features of the ultrasound image. An active contours algorithm is used at different resolution levels in order to deform onto the final boundary of the prostate capsule.

d. Accuracy evaluation of the proposed segmentation techniques is performed against boundaries extracted by a senior urologist with the same prostate ultrasound images. It is done both in 2-D with average distance between corresponding pixels on the two comparing boundaries, and in 3-D with volume difference between the two corresponding surface models reconstructed from the boundaries of prostate ultrasound images of the same patient. Refinement of the algorithms is to be performed to the steps a through c until the accuracy reaches a satisfactory level.

\section{Summary}

A new image-guided prostate biopsy system is now being developed. By using this system, the physicians will be able to make use of a global road map of the patient's prostate (the 3-D overlay display) and/or a 2-D ultrasound image display overlaid with a guidance grid color-coded with the optimized protocols. This combination of both 2-D and 3-D information will significantly enhance the physicians' situation awareness and thus lead to a substantial improved performance of cancer detection.

\section{Acknowledgements}

This research was supported in part by The Biomedical Engineering Research Grant RG-99-0115 of The Whitaker Foundation. Ariela Sofer was supported in part by NSF grant DMI-9800544. We thank Dinggang Shen for his valuable comments.

\section{References}

1. Zeng, J., Kaplan, C., Bauer, J., Sesterhenn, I., Moul, J. And Mun, S. K.: Visualization and evaluation of prostate needle biopsy. Proceedings of the Medical Image Computing and Computer Assisted Intervention Conference (1998) 285-292

2. Zeng, J., Bauer, J., Sofer, A., Yao, X., Opell, B., Zhang, W., Sestrehenn, I. A., Moul, J. W., Lynch, J., Mun, S. K.: Distribution of Prostate Cancer for Optimized Biopsy Protocols. Proceedings of the Medical Image Computing and Computer Assisted Intervention Conference (2000) 287-296

3. Sofer, A., Zeng, J., Opell, B., Bauer, J., Mun, S.K.: Optimal Biopsy Protocols for Prostate Cancer. In revision for publication in Interfaces (2001) 